\title{
Analysis of Bitcoin Prices using Deep Learning Techniques
}

\author{
P Ravindhar Reddy ${ }^{1}$, N Venkatadri ${ }^{2}$ \\ ${ }^{1} \mathrm{PG}$ Scholar,Department of Computer Science and Engineering \\ ${ }^{2}$ Professor, Department of Computer Science and Engineering \\ Teegala Krishna Reddy Engineering College, Meerpet, Hyderabad, Telangana 500097
}

\begin{abstract}
Article History:Received:11 november 2020; Accepted: 27 December 2020; Published online: 05 April 2021
Abstract -Project training - these projects are done for specific subjects without discussing methods that require knowledge and level of technical material. This paper discusses the design and implementation of project training in information technology as a major project that requires student creativity and describes the project clearly and concisely. These projects not only develop basic or technical skills but also practical skills. The goal of the modern student project is to show how trained machine models can predict cryptocurrency security if we allow appropriate levels of information and computing power. The diagram shows the approximate value. Conventional technology is way out of technology that can help people predict future events. With so much data being generated and stored daily, we are finally approaching an age where predictions are made from accurate and accurate data. Besides, with the advent of the digital currency era, more and more leaders have entered the digital investment market. This allows us to create models that can predict cryptocurrencies, especially Bitcoin. This can be done through several types of machine learning methods and in-depth learning and learning methods.
\end{abstract}

\section{Keywords:}

\section{INTRODUCTION}

In a multidisciplinary education system, learning about projects is one of the most attractive demonstration strategies to engage students in the right, practical responsibility to enhance learning. Project-based learning usually involves personal or group relationships between students and teachers, coaches, or mentors. Every project in the field of information technology develops and implements ways to understand the practical workplace. In this section, our students use project training to promote bitcoin inventory; In addition to technical science, they learn to use resources and time wisely and work in groups. Over the past decade, much effort has been put into creating algorithms and models to predict future prices and events. Because user data is collected in multiple ways, there is no such raw data at present. Every model that can predict a future event, whether it knows what the future holds or predicts future client movements, has a more predictable style and the ability to change possibilities. Unit price forecasts do not vary. For years, analysts and researchers have been trying to improve and improve algorithms that predict future prices. Unfortunately, not only is the price old, but it should be considered a major contributor, and other factors such as economic growth, general popularity, and capital also play an important role in the price. Bitcoin was the first cryptocurrency created in 2009. But it became popular in 2012. In general, the currency is only a numerical line that can have economic value. This figure was developed by a powerful computer. Since the advent of Bitcoin, many other currencies, also known as Altcoin, have emerged. Because bitcoin is not managed by any bank and is open to transactions using the Blockchain method, it is explained by the lack of tax intermediaries or research instruments. Bitcoin can be considered gold in the early 19th century. Banks and traders are willing to exchange that money for cash, but they do not have complete control over them. Similarly, bitcoin can be sold for cash, and even for sale. Each coin is mined and there are only twenty-one million coins, of which 11 million have been mined. One of the challenges faced by analysts and researchers is to implement a system that can predict the actual price. In this innovative project, our students develop machine learning algorithms to predict short-term changes in the price of bitcoin based on data from a historical sequence of numerical factors that affect the price of bitcoin. His idea is to implement a system that analyzes data in real-time and guides investors in making decisions. Applications receive data from the real world and undergo transformational datasets that prepare data entry for machine learning algorithms. With the help of this data, we can predict the price of Bitcoin tomorrow.

SCOPE

The Neural Network (RNN) developed in this project was completed using the Hard Library. Hard provides API neural networks that work with Tensorflow or Theano. Hardly selected with a user-friendly API and CPU and GPU support capabilities.Hard to run the model without any problems. Like all Python libraries, Monkey users provide modules and module modules that are automatically defined. This module can also be upgraded so that developers can create new and matching models that fit their needs. Since all numbers are written only in Python, there is no problem for Python developers to correct errors or run malicious code.

${ }^{*}$ Corresponding author: P Ravindhar Reddy

PG Scholar,Department of Computer Science and Engineering

Teegala Krishna Reddy Engineering College, Meerpet, Hyderabad, Telangana 500097 


\section{PURPOSE OF PROJECT}

Bitcoin - an electronic payment system (EMS), designed to create currency units called bitcoins into BTC. Many electronic money systems have been successful in the past, but Bitcoin is different and it is new and unique crypto that has a system that tries to reduce the cost problem for EMS. Digital currencies use currency management to require central authorities to participate in transactions, forcing them to be at risk of monitoring their foreign exchange reserves or resolving disputes. The upper limit is defined by the number of units of gold and carefully compared to smaller sources such as gold. Digital currency is a digital currency that uses privacy to support processes related to devising activity and design. Note that Bitcoin is the first cryptocurrency in the world, created in 2009 on a white paper named Satoshi Nakamoto. Therefore, the price of Bitcoin changes all the time, so we need to predict the future price of bitcoin-based on available data.

\section{LITERATURE SURVEY}

Bitcoin is an electronic money transfer system (EMS) that is also used to exchange currencies called bitcoins or BTC [6]. Many electronic money systems have been successful in the past, but Bitcoin differs in that it is new and unique crypto that has a mechanism that tries to alleviate the cost of EMS. Cryptocurrency uses cryptocurrency management to require central authorities to participate in transactions, forcing them to risk managing foreign exchange reserves or mediation disputes. The upper limit of the amount of cryptocurrency is explained and closely monitored to mimic small resources such as gold.

Cryptocurrency is a digital currency that uses cryptography to support processes related to transactions with device manufacturers. In particular, Bitcoin is the first decentralized cryptocurrency in the world, created in 2009 on a white paper coded by Satoshi Nakamoto. In the central currency, the government or other legal entity controls the supply of foreign currency by printing the new currency. Bitcoin, on the other hand, is an unknown currency, so no organization is responsible for creating new devices or bitcoin. Bitcoin offers a secure way to anonymize digital transactions. When making Bitcoin transactions, there should be a way to manage Bitcoin orders. In the last line, it is easy to know who is buying; you need to solve math puzzles. When these math puzzles are solved and transactions are sorted, a new bitcoin is created and inserted into the system. Currently, the prize for adding transactions to the Blockchain is 12.5 new bitcoin. Every bitcoin is removed from this transaction. Rewards to increase blockchain transactions twice every four years and reach around 2,140. There is currently a limit of 21 million bitcoin units in circulation. As the amount of bitcoin in the post reaches its limit, trading bitcoin becomes increasingly difficult over time. The size of bitcoin until January 2018 can be one of the reasons why bitcoin prices are high.

Bitcoin prices seem to be rising. For example, on November 15,2017 , the price was $\$ 7,854$. A month later, on December 15, 2017, the price reached a record high of $\$ 19,511$. Then, on December 28, 2017, the price dropped 27\% from that record., Bitcoin prices will range between \$6,000 and \$11,000 until February 2018. Due to such instability, it is possible to pay large amounts and lose ions. Many people in this unstable market are looking for ways and means to make money. With this in mind, the question arises as to whether Bitcoin income is calculated. This is a simple question, especially given the short history of bitcoin, as little research has been done on bitcoin income forecasts.

Many studies have seen the value of bitcoin and other cryptocurrencies. For example, the author [10] developed a model for the price of bitcoin and offered some discussions about the model's ability to explain the price of bitcoin. In [11], the author uses an equilibrium model to evaluate bitcoin and centralized torpedo assets. Several other studies have examined the influence of Blockchain and related technologies in other financial sectors. Raskin and Fair [12] examined the results at the central bank. [13] Focused on corporate governance. [14] The author studied the cost of bitcoin mining. [15] The author offers a more in-depth discussion of crypto mechanisms.

Some studies provide a complete technical analysis. [16] Many studies have looked at some aspects of mediumsized strategies in the financial markets. The work was done [17-19] aims to achieve this strategy in the security market. $[20,21]$ The MA authors formulate specific strategies and procurement and storage strategies based on specific capital coverings. [22] The study predicted significant risks incorporate prices based on technical indicators. [23] Working with ETFs and Master Assessment Strategies.

This text differs from previous research in that it was the first to predict the value of bitcoin with a complete teaching method and model that predicted 26 Django qualifications used in industrial development.

Carnegie Mellon University offers its first year of "Basic Mechanical Engineering" aimed at introducing students to mechanical engineering. The courses are aimed at stimulating students' interest in technical engineering in the early stages of their careers, integrating basic knowledge of engineering concepts, physics and mathematics, and problem-solving as major engineering activities. This goal is achieved through real-life examples, teaching classes, and practical tasks and laboratory experiments. During the eleventh semester of this course, a team of first-time students designed and installed electric transmission systems using steam engines and McCannets to activate mobile vehicles or turn off lights. The article describes the systemic process for promoting this course and the process of carefully combining lessons with class introductions, laboratory tests, and practical projects to encourage learning. 


\section{OVERVIEW OF THE SYSTEM}

\section{A. Existing System:}

Algorithms have been used successfully to support vector machines in the future, as has been done in research [24 - 28]. In particular, SVM is recommended to work well with small or volatile data and is often used to predict the return on this investment. The SVM classification has the advantage that it provides the best values in the world. In this project, the estimation model is analyzed based on the data entered and the accuracy of the results. However, there are no current proposals to make Bitcoin and SVM predictions impossible.

Disadvantages:

$>$ SVM is not suitable for TimeSeries analysis.

$>$ Wrong and inaccurate predictions.

$>$ Small functions work with small data or voice mail.

\section{B. Proposed system:}

The RNN Nervous Network (RNN) is a high-level training method developed in the late 1980s. This neural network is perfect for follow-up information. This is best because you can forget the weight in each class and move it to the next class. The RNN uses internal memory and the end cell value in the cell to record a series of data on the right side. Logs are removed and sent through three ports: Forgot Gate, Entrance Gate, and Exit Gate. The sigmoid function is performed on each door with results ranging from 0 to 1 . Thus, when we pay attention to this class, we are at a rate that changes our contribution from the kidney tissue.

Advantages:

$>$ Using Network Learning Recurring (RNN) to predict Bitcoin suitable for TimeSeries analysis.

$>$ Great work with big data

$>$ Easy to implement and provides better predictions for future Bitcoin prices.

\section{Modules Description}

Supervised Classification (Training Dataset)

Tasks are divided into two parts, namely training and test data at 70:30. Learning algorithms are based on training data and a set of experimental data is calculated based on training.

Supervised Classification (Test Dataset)

The experimental group contains $30 \%$ of all data. Guided learning methods are used for experimental data and the results compared to the actual results.

Keras:

Keras is an open source for torpedoes written in Python. It works with TensorFlow, Microsoft Identification Tool, R, Theano, or PlaidML. Designed for quick tests and a comprehensive network of friends, easy to use, modify, and apply.

\section{Recurrent Networks (RNN):}

RNN is an advanced learning technique developed in the late 1980s. This neural network is perfect for accompanying data. This is best because you can forget the weight of each category and move it to the next unit. The RNN uses internal memory and estimates the value of the right cell to store adjacent rows of data. Logs are deleted and sent through three ports: forgotten port, entry port, and exit port. The sigmoid function is applied to each door at all levels to give results from 0 to 1 . So, when we enter a value into this unit, we measure the input that is sent to the Neural Network

\section{OUTPUT SCREEN SHOTS}

\begin{tabular}{rrrrrrrrr} 
& Timestamp & Open & High & Low & Close & Volume_(BTC) & Volume_(Currency) & Weighted_Price \\
\hline 0 & 1417411980 & 300.0 & 300.0 & 300.0 & 300.0 & 0.01 & 3.0 & 300.0 \\
1 & 1417412040 & $\mathrm{NaN}$ & $\mathrm{NaN}$ & $\mathrm{NaN}$ & $\mathrm{NaN}$ & $\mathrm{NaN}$ & $\mathrm{NaN}$ & $\mathrm{NaN}$ \\
2 & 1417412100 & $\mathrm{NaN}$ & $\mathrm{NaN}$ & $\mathrm{NaN}$ & $\mathrm{NaN}$ & $\mathrm{NaN}$ & $\mathrm{NaN}$ & $\mathrm{NaN}$ \\
3 & 1417412160 & $\mathrm{NaN}$ & $\mathrm{NaN}$ & $\mathrm{NaN}$ & $\mathrm{NaN}$ & $\mathrm{NaN}$ & $\mathrm{NaN}$ & $\mathrm{NaN}$ \\
4 & 1417412220 & $\mathrm{NaN}$ & $\mathrm{NaN}$ & $\mathrm{NaN}$ & $\mathrm{NaN}$ & $\mathrm{NaN}$ & $\mathrm{NaN}$ & $\mathrm{NaN}$
\end{tabular}


<class 'pandas.core.frame.DataFra

RangeIndex: 1990691 entries, 0 tc

Data columns (total 9 columns):

Timestamp int64

Open

float 64

High

float 64

Low

float 64

Close

float 64

Volume_(BTC)

float 64

Volume_(Currency) float 64

Weighted_Price

date

float 64

dtypes: float64(7), int64(1), obj

momnny ucsas. $1367+$ MR

Epoch $3 / 10$

1434/1434 [ $===========================$ ] - Os 174us/step - loss : 0.0260

Epoch $4 / 10$

1434/1434 [ $============================$ ] - Os 175us/step - loss: 0.0212

Epoch $5 / 10$

1434/1434 [ $============================$ ] - Os 175us/step - loss: 0.0164

Epoch $6 / 10$

1434/1434 [ $[=============================$ ] - Os 176us/step - loss: 0.0117

Epoch $7 / 10$

1434/1434 [ $=========================$ ] - Os 180us/step - loss: 0.0075

Epoch 8/10

1434/1434 [ $==========================$ ] - Os 185us/step - loss: 0.0042

Epoch $9 / 10$

1434/1434 [ $==========================$ ] - OS 177us/step - loss: 0.0020

ramele 1s/1s
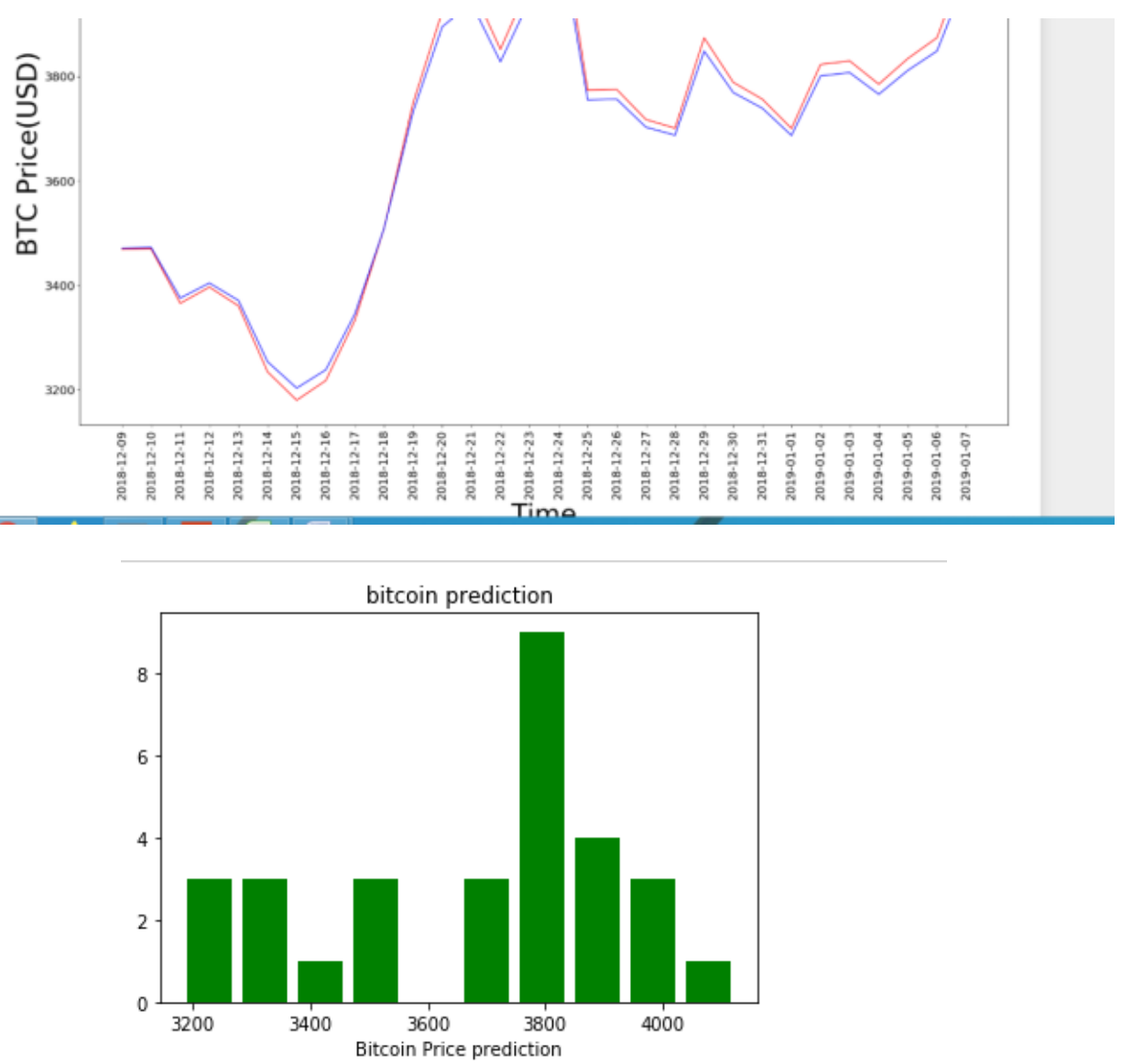


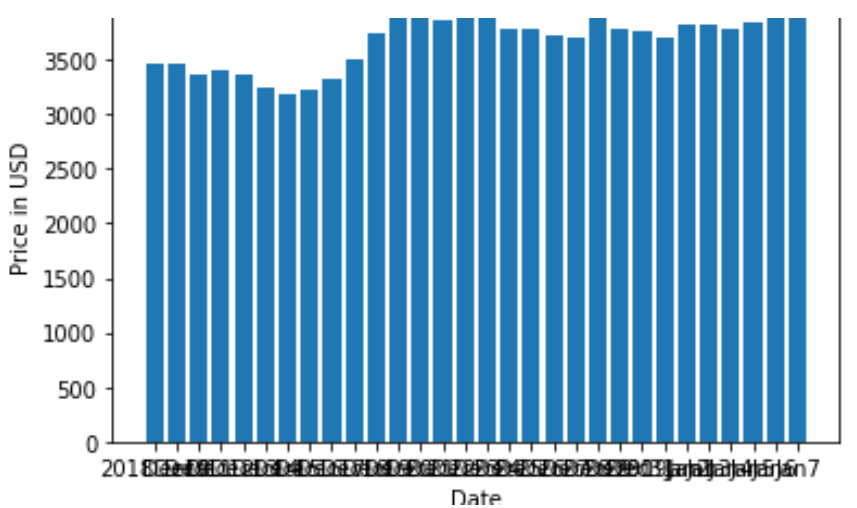

\section{CONCLUSION AND FUTURE SCOPE \\ CONCLUSION}

Looking ahead is always at the top of the list of machine learning algorithms. Here, in this project, we try to predict the price of bitcoin by two in-depth training methods. This thesis is intended to provide project training in information technology, as well as problem recognition, success, student assessment, and practical training applications based on the use of comprehensive algorithms to create software. You can predict the price of Bitcoin.

Django can implement web applications that show a complete picture of learning. However, persuasive nerve cell time is designed primarily for the study and distribution of images and numerical predictions. Although the buffer size is not only 5\% required, it can compete with the LSTM model. Thanks to this project, students can learn and understand all life cycles in the program. They also have experience with Django in the development of machine learning methods and network development. Predictive models will be more complex and effective in the future, the amount of data collected will increase and strategies to improve data analysis will be developed. The only reason we are limited is the increase in arithmetic power. There are always opportunities for improvement and with advanced science, these improvements are possible: - Learn a better database model to improve prediction accuracy. - Model a large number of neurons and assign them to a computer or small system group. - Add more services to the item map and connect your model with other types so that customers can learn from specific products according to their needs.

\section{FUTURE ENHANCEMENTS}

It is important to take a holistic approach to forecast, as it requires further research to look for other promising services. The use of data in linking links with data samples to predict outcomes can be divided into repetitive models or K-source groups. These common data points can be used in a comprehensive knowledge model. New students can select some topics from the research comments above.

\section{REFERENCES}

G. Solomon, "Project-Based Learning: A Primer," Technology Learning, volume 23, Jan. 2003.

M. Hedley, "An undergraduate microcontroller systems laboratory," IEEE Transactions in Education, vol. 41(4), pp. 345-353, Nov. 1998.

H. Markkanen, G. Donzellini, and D. Ponta, "NetPro: Methodologies and tools for project-based learning on the internet," in Proceedings of World Conference on Educational Multimedia, pp. 1230-1235.

D. Ponta, G. Donzellini, and H. Markkanen, "NetPro: Network-based project learning on the internet," in Proceedings of European Symposium of Intelligent Technologies, pp. 703-708, 2002.

S. A. Ambrose and C. H. Amon, "Systematic design of a first-year mechanical engineering course at CarnegieMellon University," Journal of Engineering Education, vol. 86, pp. 173-182, Apr. 1997.

"Vocabulary - Bitcoin." [Online]. Available: https://bitcoin.org/en/vocabulary\#btc.

"PayPal." [Online]. Available: http://en.wikipedia.org/wiki/PayPal.

Nakamoto, S. (2008). Bitcoin: A peer-to-peer electronic cash system. https://bitcoin.org/bitcoin.pdf

Harvey, C. (2014). Bitcoin myths and facts.Working paper, Duke University. Available at http://ssrn.com/abstract $=2479670$

Athey, S., I. Parashkevov, V. Sarukkai, and J. Xia (2016). Bitcoin pricing, adoption, and usage: Theory and evidence. Working paper, Stanford University. 